\title{
ADVANCED DEEP LEARNING MODEL
}

\author{
Yew Kee Wong \\ School of Information Engineering, HuangHuai University, Henan, China
}

\begin{abstract}
Deep learning is a type of machine learning that trains a computer to perform human-like tasks, such as recognizing speech, identifying images or making predictions. Instead of organizing data to run through predefined equations, deep learning sets up basic parameters about the data and trains the computer to learn on its own by recognizing patterns using many layers of processing. This paper aims to illustrate some of the different deep learning algorithms and methods which can be applied to artificial intelligence analysis, as well as the opportunities provided by the application in various decision making domains.
\end{abstract}

\section{KEYWORDS}

Artificial Intelligence, Machine Learning, Deep Learning.

\section{INTRODUCTION}

Deep learning is one of the foundations of artificial intelligence (AI), and the current interest in deep learning is due in part to the buzz surrounding AI. Deep learning techniques have improved the ability to classify, recognize, detect and describe - in one word, understand [1]. For example, deep learning is used to classify images, recognize speech, detect objects and describe content.

Several developments are now advancing deep learning:

- Algorithmic improvements have boosted the performance of deep learning methods.

- New machine learning approaches have improved accuracy of models.

- New classes of neural networks have been developed that fit well for applications like text translation and image classification.

- We have a lot more data available to build neural networks with many deep layers, including streaming data from the Internet of Things, textual data from social media, physicians notes and investigative transcripts.

- Computational advances of distributed cloud computing and graphics processing units have put incredible computing power at our disposal. This level of computing power is necessary to train deep algorithms.

At the same time, human-to-machine interfaces have evolved greatly as well. The mouse and the keyboard are being replaced with gesture, swipe, touch and natural language, ushering in a renewed interest in AI and deep learning [2]. This paper will look at some of the different deep learning algorithms and methods which can be applied to AI analysis, as well as the opportunities provided by the application in various decision making domains. 


\section{HoW DEEP LEARNING WORKS}

Deep learning changes how you think about representing the problems that you are solving with analytics. It moves from telling the computer how to solve a problem to training the computer to solve the problem itself.

A traditional approach to analytics is to use the data at hand to engineer features to derive new variables, then select an analytic model and finally estimate the parameters (or the unknowns) of that model. These techniques can yield predictive systems that do not generalize well because completeness and correctness depend on quality of the model and its features [3]. For examples, if you develop a fraud model with feature engineering, you start with a set of variables, and you most likely derive a model from those variables using data transformations. You may end up with 30,000 variables that your mode depends on, then you have to shape the model, figure out which variables are meaningful, which ones are not, and so on. Adding mode data requires you to do it all over again.

The new approach with deep learning is to replace the formulation and specification of the model with hierarchical characterizations (or layers) that learn to recognize latent features of the data from the regularities in the layers. The paradigm shift with deep learning is a move from feature engineering to feature representation. The promise of deep learning is that it can lead to predictive systems that generalize well, adapt well, continuously improve as new data arrives, and are more dynamic than predictive systems built on hard business rules. You no longer fit a model. Instead, you train the task.

Deep learning is making a big impact across industries. In life sciences, deep learning can be used for advanced image analysis, research, drug discovery, prediction of health problems and disease symptoms, and the acceleration of insights from genomic sequencing. In transportation, it can help autonomous vehicles adapt to changing conditions [4[. It is also used to protect critical infrastructure and speed response.

More deep learning methods use neural networks architectures, which is why deep learning models are often referred to as deep neural networks. The term "deep" usually refers to the number of hidden layers in the neural network. Traditional neural networks only contain 2-3 hidden layers, while deep networks can have as many as 150. Deep learning models are trained by using large sets of labelled data and neural network architectures that learn features directly from the data without the need for manual feature extraction.

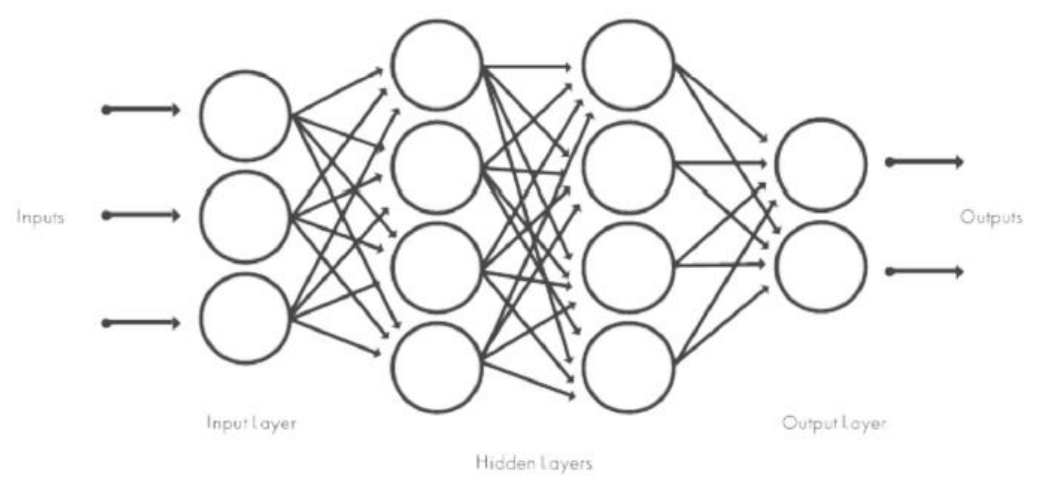

Figure 1: Neural networks, which are organized in layers consisting of a set of inter connected nodes. Networks can have tens or hundreds of hidden layers. 


\section{How DeEp Learning BEING USED}

To the outside eye, deep learning may appear to be in a research phase as computer science researchers and data scientists continue to test its capabilities. However, deep learning has many practical applications that businesses are using today, and many more that will be used as research continues [5]. Popular uses today include:

\section{Speech Recognition}

Both the business and academic worlds have embraced deep learning for speech recognition. Xbox, Skype, Google Now and Apple's Siri ${ }^{\circledR}$, to name a few, are already employing deep learning technologies in their systems to recognize human speech and voice patterns.

\section{Natural Language Processing}

Neural networks, a central component of deep learning, have been used to process and analyse written text for many years. A specialization of text mining, this technique can be used to discover patterns in customer complaints, physician notes or news reports, to name a few.

\section{Image Recognition}

One practical application of image recognition is automatic image captioning and scene description. This could be crucial in law enforcement investigations for identifying criminal activity in thousands of photos submitted by bystanders in a crowded area where a crime has occurred. Self-driving cars will also benefit from image recognition through the use of 360degree camera technology.

\section{Recommendation Systems}

Amazon and Netflix have popularized the notion of a recommendation system with a good chance of knowing what you might be interested in next, based on past behaviour. Deep learning can be used to enhance recommendations in complex environments such as music interests or clothing preferences across multiple platforms.

Recent advances in deep learning have improved to the point where deep learning outperforms humans in some tasks like classifying objects in images [6]. While deep learning was first theorized in the 1980s, there are two main reasons it has only recently become useful:

1. Deep learning requires large amounts of labelled data. For example, driverless car development requires millions of images and thousands of hours of video.

2. Deep learning requires substantial computing power. High-performance GPUs have a parallel architecture that is efficient for deep learning. When combined with clusters or cloud computing, this enables development teams to reduce training time for a deep learning network from weeks to hours or less.

When choosing between machine learning and deep learning, consider whether you have a highperformance GPU and lots of labelled data. If you don't have either of those things, it may make more sense to use machine learning instead of deep learning. Deep learning is generally more complex, so you'll need at least a few thousand images to get reliable results. Having a highperformance GPU means the model will take less time to analyse all those images [7]. 


\section{DEEP LEARNING OPPORTUNITIES AND APPLICATIONS}

A lot of computational power is needed to solve deep learning problems because of the iterative nature of deep learning algorithms, their complexity as the number of layers increase, and the large volumes of data needed to train the networks.

The dynamic nature of deep learning methods - their ability to continuously improve and adapt to changes in the underlying information pattern - presents a great opportunity to introduce more dynamic behaviour into analytics [8]. Greater personalization of customer analytics is one possibility. Another great opportunity is to improve accuracy and performance in applications where neural networks have been used for a long time. Through better algorithms and more computing power, we can add greater depth.

While the current market focus of deep learning techniques is in applications of cognitive computing, there is also great potential in more traditional analytics applications, for example, time series analysis. Another opportunity is to simply be more efficient and streamlined in existing analytical operations. Recently, some study showed that with deep neural networks in speech-to-text transcription problems [9]. Compared to the standard techniques, the worderrorrate decreased by more than 10 percent when deep neural networks were applied. They also eliminated about 10 steps of data preprocessing, feature engineering and modelling. The impressive performance gains and the time savings when compared to feature engineering signify a paradigm shift.

Here are some examples of deep learning applications are used in different industries:

Automated Driving: Automotive researchers are using deep learning to automatically detect objects such as stop signs and traffic lights. In addition, deep learning is used to detect pedestrians, which helps decrease accidents.

Aerospace and Defence: Deep learning is used to identify objects from satellites that locate areas of interest, and identify safe or unsafe zones for troops.

Medical Research: Cancer researchers are using deep learning to automatically detect cancer cells. Teams at UCLA built an advanced microscope that yields a high-dimensional data set used to train a deep learning application to accurately identify cancer cells [10].

Industrial Automation: Deep learning is helping to improve worker safety around heavy machinery by automatically detecting when people or objects are within an unsafe distance of machines.

Electronics: Deep learning is being used in automated hearing and speech translation. For example, home assistance devices that respond to your voice and know your preferences are powered by deep learning applications.

\section{How to Create and Train Deep Learning Models}

The three most common ways people use deep learning to perform object classification are:

\section{Training from Scratch}

To train a deep network from scratch, you gather a very large labelled data set and design a network architecture that will learn the features and model. This is good for new applications, or 
applications that will have a large number of output categories. This is a less common approach because with the large amount of data and rate of learning, these networks typically take days or weeks to train [11].

\section{Transfer Learning}

Most deep learning applications use the transfer learning approach, a process that involves finetuning a pre-trained model. User can start with an existing network, such as AlexNet or GoogLeNet, and feed in new data containing previously unknown classes [12]. After making some tweaks to the network, user can now perform a new task, such as categorizing only dogs or cats instead of 10,000 different objects. This also has the advantage of needing much less data (processing thousands of images, rather than millions), so computation time drops to minutes or hours.

\section{Feature Extraction}

A slightly less common, more specialized approach to deep learning is to use the network as a feature extractor. Since all the layers are tasked with learning certain features from images, user can pull these features out of the network at any time during the training process [13]. These features can then be used as input to a machine learning model such as support vector machines (SVM).

\section{Conclusions}

So this study was concerned by understanding the interrelation between deep learning and AI, what frameworks and systems that worked, and how deep learning can impact the learning process whether by introducing new innovations that foster advanced deep learning process and escalating power consumption, security issues and replacing human in workplaces [14]. The advanced deep learning algorithms with various applications show promising results in artificial intelligence development and further evaluation and research using smarter deep learning models are in progress.

\section{REFERENCES}

[1] Shi, Z., (2019). Cognitive Machine Learning. International Journal of Intelligence Science, 9, pp. 111-121.

[2] Lake, B.M., Salakhutdinov, R. and Tenenbaum, J.B., (2019). Human-Level Concept Learning through Probabilistic Program Induction. Science, 350, pp. 1332-1338.

[3] Silver, D., Huang, A., Maddison, C.J., et al., (2016). Mastering the Game of Go with Deep Neural Networks and Tree Search. Nature, 529, pp. 484-489.

[4] Fukushima, K., (1980). Neocognitron: A Self-Organizing Neural Network Model for a Mechanism of Pattern Recognition Unaffected by Shift in Position. Biological Cybernetics, 36, pp. 193-202.

[5] Lecun, Y., Bottou, L., Orr, G.B., et al., (1998). Efficient Backprop. Neural Networks Tricks of the Trade, 1524, pp. 9-50.

[6] McClelland, J.L., et al., (1995). Why There Are Complementary Learning Systems in the Hippocampus and Neocortex: Insights from the Successes and Failures of Connectionist Models of Learning and Memory. Psychological Review, 102, pp. 419-457.

[7] Kumaran, D., Hassabis, D. and McClelland, J.L., (2016). What Learning Systems Do Intelligent Agents Need? Complementary Learning Systems Theory Updated. Trends in Cognitive Sciences, 20, pp. 512-534.

[8] Wang, R., (2019). Research on Image Generation and Style Transfer Algorithm Based on Deep Learning. Open Journal of Applied Sciences, 9, pp. 661-672. 
[9] Krizhevsky, A., Sutskever, I., Hinton, G.E., et al., (2012). ImageNet Classification with Deep Convolutional Neural Networks. Neural Information Processing Systems, 141, pp. 1097-1105.

[10] Long, J., Shelhamer, E., Darrell, T., et al., (2015). Fully Convolutional Networks for Semantic Segmentation. Computer Vision and Pattern Recognition, Boston, pp. 3431-3440.

[11] Noh, H., Hong, S., Han, B., et al., (2015). Learning Deconvolution Network for Semantic Segmentation. International Conference on Computer Vision, Santiago, 7-13, pp. 1520-1528.

[12] Cheng, Z., Yang, Q., Sheng, B., et al., (2015). Deep Colorization. International Conference on Computer Vision, Santiago, pp. 415-423.

[13] Mahendran, A. and Vedaldi, A., (2015). Understanding Deep Image Representations by Inverting Them. Computer Vision and Pattern Recognition, Boston, pp. 5188-5196.

[14] Gatys, L.A., Ecker, A.S. and Bethge, M., (2015). Texture Synthesis Using Convolutional Neural Networks. In: Advances in Neural Information Processing Systems, Neural Information Processing Systems Foundation, Quebec, pp. 262-270.

\section{AUTHOR}

Prof. Yew Kee Wong (Eric) is a Professor of Artificial Intelligence (AI) \& Advanced Learning Technology at the HuangHuai University in Henan, China. He obtained his BSc (Hons) undergraduate degree in Computing Systems and a Ph.D. in AI from The Nottingham Trent University in Nottingham, U.K. He was the Senior Programme Director at The University of Hong Kong (HKU) from 2001 to 2016. Prior to joining the education sector, he has worked in international technology companies, HewlettPackard (HP) and Unisys as an AI consultant.

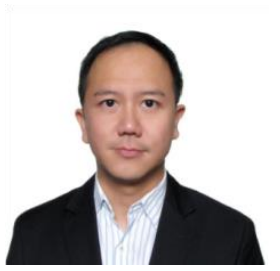
His research interests include AI, online learning, big data analytics, machine learning, Internet of Things (IOT) and blockchain technology.

(C) 2021 By AIRCC Publishing Corporation. This article is published under the Creative Commons Attribution (CC BY) license. 